\title{
Optical sinc-shaped Nyquist pulses with very low roll-off generated from a rectangular frequency comb
}

\author{
Marcelo A. Soto ${ }^{1, *}$, Mehdi Alem ${ }^{1}$, Mohammad Amin Shoaie ${ }^{2}$, Armand Vedadi ${ }^{2}$, Camille-Sophie Brès ${ }^{2}$, \\ Thomas Schneider ${ }^{3}$, and Luc Thévenaz ${ }^{1}$ \\ ${ }^{1}$ EPFL Swiss Federal Institute of Technology, Group for Fibre Optics (GFO), SCI-STI-LT, Station 11, CH-1015 Lausanne, Switzerland \\ ${ }^{2}$ EPFL Swiss Federal Institute of Technology, Photonic Systems Laboratory (PHOSL), SCI-IEL, Station 11, CH-1015 Lausanne, Switzerland \\ ${ }^{3}$ Institut für Hochfrequenztechnik, Hochschule für Telekommunikation Leipzig, Gustav-Freytag-Str. 43-45, 04277 Leipzig, Germany \\ *Email: marcelo.soto@epfl.ch
}

\begin{abstract}
High-quality optical sinc-shaped Nyquist pulses with a virtual zero roll-off factor are obtained from the generation of a phase-locked, rectangular-spectrum frequency comb. Tunability over more than 4 frequency decades is demonstrated with $<1 \%$ power distortion.

OCIS codes: (060.4510) Optical communications; (060.4080) Modulation; (320.5540) Pulse shaping.
\end{abstract}

\section{Introduction}

The continuous increase in the demand for higher data transmission rates has motivated the research of new approaches to improve the spectral efficiency of optical communication networks [1]. In principle, the data transmission rates can be increased using [1,2]: i) ultra-short pulses per channel, ii) higher-order modulation formats, and iii) orthogonal multiplexing with high spectral efficiency. While ultra-short pulses are highly susceptible to chromatic dispersion (CD) and polarization mode dispersion (PMD), higher-order modulation formats require higher optical signal-to-noise ratio as well as very sophisticated electrical digital signal processors (DSP) [1]. High-spectral efficiency can be achieved by orthogonal frequency division multiplexing (OFDM) or Nyquist pulses transmission [2], enabling ultrahigh data transmission rates and a simultaneous ultrahigh spectral efficiency.

Nyquist pulses can be orthogonally multiplexed in a wavelength division multiplexing scheme so-called Nyquist- Wavelength-division multiplexing (WDM) [3], or in a time division multiplexing scheme so-called orthogonal TDM [4]. While orthogonal TDM can communicate most reliably by use of Nyquist pulses with minimum inter-symbol interference (ISI), a spectrally-efficient Nyquist-WDM transmission requires no guard band between adjacent frequency channels. These two independent properties can only be achieved simultaneously by sinc-shaped Nyquist pulses [5]. Combining several WDM channels, each of which including many orthogonal TDM channels, makes possible the transmission of $\mathrm{Tb} / \mathrm{s}$ superchannel, which can be routed through the existing optical networks as a single entity. Using sinc-shaped Nyquist pulses such an aggregation of channels can be generated with full spectral efficiency and with temporally zero ISI, therefore minimizing transmission errors.

In this paper, optical sinc-shaped Nyquist pulses with unprecedented high quality are obtained based on the generation of a phase-locked frequency comb with an ideal rectangular spectral shape. The wide tunability of the method using two cascaded electro-optic intensity modulators is experimentally demonstrated over 4 frequency decades. To evaluate the pulse spectral broadening resulting from modulation, these high-quality generated sincshaped Nyquist pulses are modulated with on-off keying (OOK) and binary phase-shift keying (BPSK) formats. Modulated sinc pulses with roll-off factor equal to 0.11 are reported.

\section{Proposed method for sinc-shaped Nyquist pulses}

In communication systems, data can be distorted when symbols interfere with their neighbors, resulting in the socalled inter-symbol interference (ISI) [5]. This unwanted phenomenon induces errors in signal detection and makes the communication less reliable. ISI can be minimized by shaping data pulses according to the so-called Nyquist criterion [5], in which the pulse $x(t)$ is equal to 0 for all sampling time $n \tau$ (where $n$ is an integer and $\tau$ is the sampling period), except at time origin where $x(0)=1$. Raised-cosine pulses [4,5] satisfy the Nyquist ISI criterion, and are among the most used pulses in telecommunications.

$$
x(t)=\operatorname{sinc}\left(\frac{t}{\tau}\right) \frac{\cos (\alpha \pi t / \tau)}{1-(2 \alpha t / \tau)^{2}}
$$

where $\alpha(0 \leq \alpha \leq 1)$ is the roll-off factor [4,5], which is a measure of the pulse excess bandwidth with respect to the ideal sinc pulse, represented by $\alpha=0$ [5]. Thus, while the bandwidth of raised-cosine pulses is $(1+\alpha) / \tau$, sinc pulses $\operatorname{sinc}(t / \tau)$ minimize the bandwidth usage down to $1 / \tau$. Therefore, among all ISI-free pulses, sinc pulses can be exploited in wavelength-division multiplexing (WDM) systems to make an efficient usage of bandwidth and avoid any guard bands between adjacent WDM channels. 
In the Fourier representation, a sinc pulse corresponds to a rectangular spectral function, and therefore, a periodic train of sinc pulses corresponds to a rectangular-shaped and phase-locked frequency comb [6,7]. In this paper, a method to obtain sinc-shaped Nyquist pulses from the direct generation of a rectangular-shaped optical frequency comb is proposed, reaching a waveform of unprecedented quality. Here this frequency comb is generated cascading two Mach-Zehnder modulators (MZMs) driven by well-defined frequencies [6]; this results in a highly flexible method which offers the possibility of easily modifying the pulse duration and pulse repetition period by simply changing the RF modulating frequencies [7].

\section{Experimental setup}

Sequence of since-shaped Nyquist pulses are generated using the experimental setup shown in Fig. 1. Rectangular frequency combs with 9 lines are obtained using an external cavity laser (ECL) operating at $1550 \mathrm{~nm}$ and two cascaded Mach-Zehnder modulators [6]. Two synchronized microwave generators are used to drive the modulators with frequencies $f_{1}$ and $f_{2}=3 f_{1}$, respectively. The relative phase among both $\mathrm{RF}$ signals has been carefully adjusted to reach almost ideal and symmetric sinc-shaped Nyquist pulses. In order to increase the SNR of the pulses, a semiconductor optical amplifier (SOA) and an EDFA have been used in combination with two tunable optical filters (TOF). A 500-GHz optical sampling oscilloscope is used to acquire the pulse waveforms and evaluate the jitter and SNR of the generated pulses. It is worth mentioning that the optical filters are here used only to filter out the amplified spontaneous emission (ASE) noise generated by the optical amplifiers and are not employed for spectral shaping. A third modulator is then used to encode data onto ideal sinc-shaped Nyquist pulses and to evaluate the impact of spectral broadening in this case. The spectrum resulting from modulating the sinc pulses is measured using an optical spectrum analyzer (OSA) with a resolution bandwidth of $0.01 \mathrm{~nm}$.

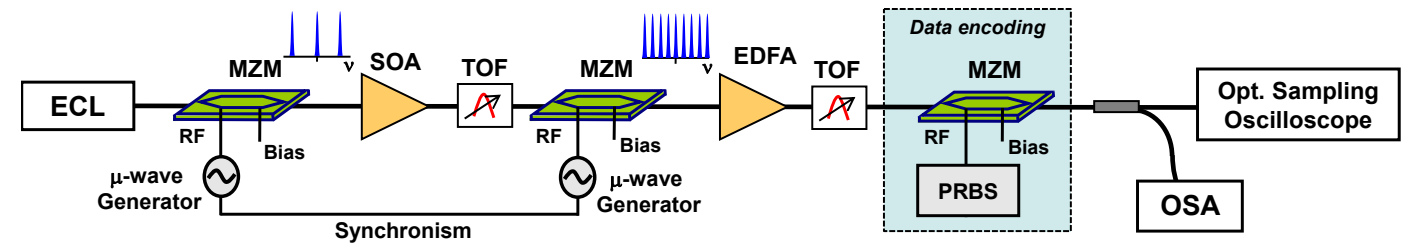

Fig. 1. Experimental setup for high-quality sinc-shaped Nyquist pulse generation based on a rectangular frequency comb.

\section{Experimental results and discussion}

The wide tunability of the proposed method has been verified by changing the RF modulating frequencies over 4 orders of magnitude. First, modulating the first and second EOM with RF signals at $f_{1}=10 \mathrm{MHz}$ and $f_{2}=30 \mathrm{MHz}$, respectively, a comb with 9 spectral components separated by $\Delta f=10 \mathrm{MHz}$, and expanding over a bandwidth of $90 \mathrm{MHz}$, has been generated. In time domain, this comb resulted in a sequence of sinc-shaped Nyquist pulses with a zero-crossing duration of $22.22 \mathrm{~ns}$, which is equivalent to a FWHM width of $9.8 \mathrm{~ns}$, and a repetition period of 100 ns, as shown in Fig 2(a). Then the spectral line separation $\Delta f$, and thus the entire bandwidth of the comb, have been easily modified by changing the frequency of the modulating signals [7]. Increasing the modulation frequencies sequentially by one order of magnitude, pulses with FWHM duration of $980 \mathrm{ps,}$ $98 \mathrm{ps}$ and $9.8 \mathrm{ps}$ have been obtained, as reported in Figs. 2(b)-(d). The pulse repetition period in each case is $10 \mathrm{~ns}$, $1 \mathrm{~ns}$, and $100 \mathrm{ps,}$ respectively, as indicated in the figures. It is remarkable that the pulses show fully similar perfect sinc shapes over the 4 decades. The jitter and SNR in all analyzed cases resulted to be below $82 \mathrm{fs}$ and more than $40 \mathrm{~dB}$, respectively.

The RMS error between measured and theoretical shapes (given by Eq. (1),
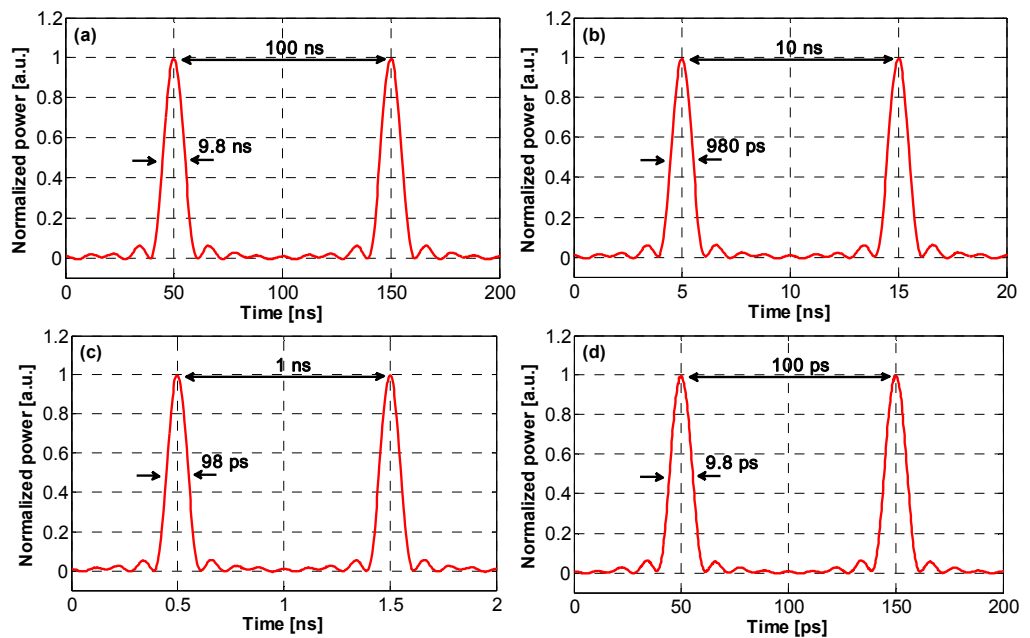

Fig. 2. sinc-shaped Nyquist pulses, obtained from frequency combs having 9 phase-locked lines spanning over a bandwidth between $90 \mathrm{MHz}$ and $90 \mathrm{GHz}$, and using (a) $f_{1}=10 \mathrm{MHz}$ and $f_{2}=30 \mathrm{MHz}$, (b) $f_{1}=100 \mathrm{MHz}$ and $f_{2}=300 \mathrm{MHz}$, (c) $f_{1}=1 \mathrm{GHz}$ and $f_{2}=3 \mathrm{GHz}$, and (d) $f_{1}=10 \mathrm{GHz}$ and $f_{2}=30 \mathrm{GHz}$. 
using $\alpha=0$ ) has been evaluated as a function of the roll-off factor $\alpha$. It has been found that in all the analyzed cases, the RMS error is minimized with a roll-off $\alpha=0$, indicating that all the measured waveforms fit very well with the theoretical ideal sinc shape (with an RMS error below 1\%), as reported in Fig. 3 for the case of using $\Delta f=10 \mathrm{GHz}$ and a full bandwidth of $90 \mathrm{GHz}$.

The $90 \mathrm{GHz}$ bandwidth frequency comb that has been so generated is plotted in Fig. 4(a). The corresponding time domain waveform actually matches the sinc pulses previously shown in Fig. 2(d). Then, data has been encoded onto such 9.8-ps sinc-shaped pulses using a pseudo-random binary sequence (PRBS) with length $2^{31}-1$. Figs. 4(b) and 4(c) show the measured spectra resulting from modulating the obtained sinc pulses with OOK and BPSK modulation formats, respectively. As expected, the spectrum of the original frequency comb broadens as a result of the modulation; however, considering that about $99 \%$ of the power still remains within the main spectral lobe, the resulting broadening is evaluated to be only $11 \%$. This corresponds to a roll-off factor equal to $\alpha=1 / N=0.11$ ( $N=9$ being the number of lines in the comb), which is significantly lower than the one obtained by other optical pulse shaping methods, reporting values up to $\alpha=0.5$ [5], even for unmodulated pulses. Since ideal sinc pulses maintain the information within the ideal Nyquist bandwidth, the spectral broadening reported here can be still reduced considerably if the number of lines in the comb is increased. For instance, using two RF signals to drive one of the EOMs, a frequency comb having $N=15$ lines can be generated, resulting in an improved roll-off factor down to $\alpha=0.067$.
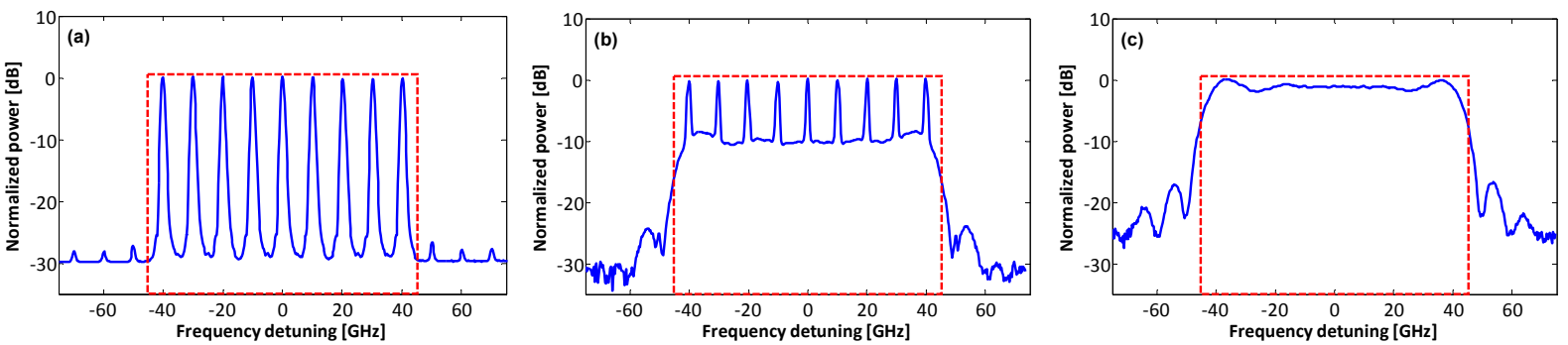

Fig. 4. Measured spectrum of (a) unmodulated sinc pulses, (b) modulated pulses with OOK format, and (c) modulated pulses with BPSK format.

\section{Conclusion}

In conclusion, a train of sinc-shaped Nyquist pulses of unprecedented high quality has been generated by a broadly tunable method based on a frequency comb generation. A zero roll-off factor, low jitter and high SNR have been verified, demonstrating the capabilities of the proposed method. The effect of data modulation on the pulse spectrum has been experimentally verified for OOK and BPSK modulation format, leading to a very low spectral broadening in comparison to other reported optical pulse shaping methods.

\section{References}

[1] C. Schubert et al., "New Trends and Challenges in Optical Digital Transmission Systems," in European Conference and Exhibition on Optical Communication (ECOC-2012) (Optical Society of America, Washington, DC, 2012), paper We.1.C.1.

[2] R. Schmogrow et al., "Real-time Nyquist pulse generation beyond $100 \mathrm{Gbit} / \mathrm{s}$ and its relation to OFDM," OpEx. 20, 317-337 (2012).

[3] D. Hillerkuss et. al., "Single-Laser 32.5 Tbit/s Nyquist WDM Transmission," Opt. Commun. Netw. 4, 715-723 (2012).

[4] M. Nakazawa et al., "Ultrahigh-speed "orthogonal” TDM transmission with an optical Nyquist pulse train," Opt. Express 20, $1129-1140$ (2012)

[5] J. G. Proakis, and M. Salehi, Digital Communications. Fifth Edition, McGraw-Hill, 2008.

[6] M. A. Soto et al., "Generation of Nyquist sinc pulses using intensity modulators," in CLEO: 2013, OSA Technical Digest (online) (Optical Society of America, 2013), paper CM4G.3.

[7] M. A. Soto et al., "Highly tunable method to generate sinc-shaped Nyquist pulses from a rectangular frequency comb," in Advanced Photonics 2013, OSA Technical Digest (online) (Optical Society of America, 2013), paper SPT2D.6. 